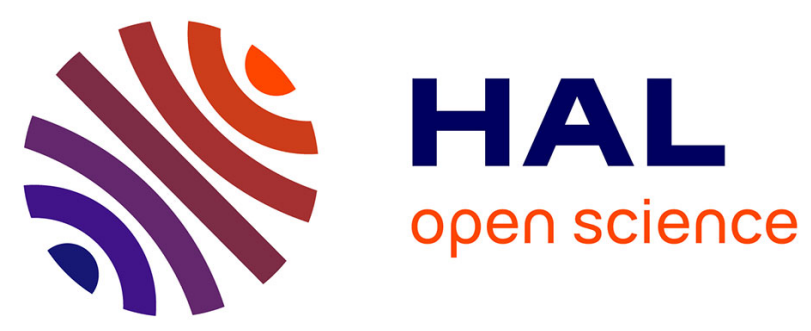

\title{
How contact interactions may depend on the shape of Cauchy cuts in N-th gradient continua: approach "à la D'Alembert"
}

Francesco Dell'Isola, Pierre Seppecher, Angela Madeo

\section{- To cite this version:}

Francesco Dell'Isola, Pierre Seppecher, Angela Madeo. How contact interactions may depend on the shape of Cauchy cuts in N-th gradient continua: approach "à la D'Alembert". Zeitschrift für Angewandte Mathematik und Physik, 2012, 63 (6), pp.1119-1141. hal-00662376

\section{HAL Id: hal-00662376 https://hal.science/hal-00662376}

Submitted on 23 Jan 2012

HAL is a multi-disciplinary open access archive for the deposit and dissemination of scientific research documents, whether they are published or not. The documents may come from teaching and research institutions in France or abroad, or from public or private research centers.
L'archive ouverte pluridisciplinaire HAL, est destinée au dépôt et à la diffusion de documents scientifiques de niveau recherche, publiés ou non, émanant des établissements d'enseignement et de recherche français ou étrangers, des laboratoires publics ou privés. 


\title{
How contact interactions may depend on the shape of Cauchy cuts in N-th gradient continua: approach "à la D'Alembert"
}

\author{
Francesco dell'Isola \\ DISG, Università di Roma "La Sapienza", via Eudossiana 18, 00184, Rome, Italy. francesco.dellisola@uniroma1.it \\ and International Research Center M\&MoCS, Palazzo Caetani, Cisterna di Latina, Latina, Italy. \\ Pierre Seppecher \\ IMath, Université du Sud-Toulon-Var, Avenue de l'Université, BP 132, 83957 La Garde Cedex, Toulon, France \\ and International Research Center M\&MoCS, Palazzo Caetani, Cisterna di Latina, Latina, Italy. \\ Angela Madeo \\ LGCIE, Université de Lyon INSA, 20 Avenue Albert Einstein, 69621 Villeurbanne CEDEX, France \\ and International Research Center M\&MoCS, Palazzo Caetani, Cisterna di Latina, Latina, Italy.
}

January 23, 2012

\begin{abstract}
Navier-Cauchy format for Continuum Mechanics is based on the concept of contact interaction between subbodies of a given continuous body. In this paper it is shown how -by means of the Principle of Virtual Powers- it is possible to generalize Cauchy representation formulas for contact interactions to the case of $\mathrm{N}$-th gradient continua, i.e. continua in which the deformation energy depends on the deformation Green-Saint-Venant tensor and all its N-1 order gradients. In particular, in this paper the explicit representation formulas to be used in $\mathrm{N}$-th gradient continua to determine contact interactions as functions of the shape of Cauchy Cuts are derived. It is therefore shown that i) these interactions must include edge (i.e. concentrated on curves) and wedge (i.e. concentrated on points) interactions, and ii) these interactions cannot reduce simply to forces: indeed the concept of K-forces (generalizing similar concepts introduced by Rivlin, Mindlin, Green and Germain) is fundamental and unavoidable in the theory of $\mathrm{N}$-th gradient continua.
\end{abstract}

\section{Introduction}

The Principle of Virtual Powers (or Virtual Works) is very ancient (see e.g. [131] and has been always considered by those French mechanicians (see e.g. [59], [60], [61], [62], [110], [29], [30], [84], [87], [88] ) who follow the ideas of D'Alembert and Lagrange as the basic concept in mechanics. More recently we can witness to a revival of the Principle of Virtual Powers: many authors (see e.g. [68], [69], [57], [58], [67], [106], [107], [108], [51], [52], [53]) finally recognize its fundamental role also in Continuum Mechanics. Actually the Principle of Virtual Powers (which can be regarded as a generalization of the Principle of Least Action, also when this last principle takes the form chosen by Hamilton and Rayleigh) has always been considered the most suitable conceptual basis for Continuum Mechanics: indeed this point of view, which maybe can be tracked up to Lagrange in his studies of fluid mechanics, has been used systematically in the famous works of E. and F. Cosserat and developed by Mindlin, Green, Rivlin, Toupin, Germain and many others (see references). A comprehensive study of this subject can be found, in particular, in a recent book, see [11]. More detailed historical studies would be required to describe how and why the importance of the Principle of Virtual Works has been underestimated for long periods in the literature: the reader will find some very interesting considerations in [88], [8], [36]. 
In this paper it has been espoused the D'Alembertian view of mechanics as explicitly stated in the foreword of the Traité de Dynamique (1768)

"La certitude des Mathématiques est un avantage que ces Sciences doivent principalement à la simplicité de leur objet. [....] les notions les plus abstraites, celles que le commun des hommes regarde comme les plus inaccessibles, sont souvent celles qui portent avec elles une plus grande lumiere: [.....] pour traiter suivant la meilleure Méthode possible [...] quelque Science que ce puisse être il est nécessaire [...] d'envisager, de la maniere la plus abstraite et la plus simple qu'il se puisse, l'objet particulier de cette Science; de ne rien supposer, ne rien admettre dans cet objet, que les propriétés que la Science même qu'on traite y suppose.

Delà résultent deux avantages: les principes reçoivent toute la clarté dont ils sont susceptibles : ils se trouvent d'ailleurs réduits au plus petit nombre possible [...]

puisque l'objet d'une Science étant nécessairement déterminé, les principes en sont d'autant plus féconds, qu'ils sont en plus petit nombre. [...]

La Méchanique surtout, est celle qu'il paroit qu'on a négligée le plus à cet égard: aussi la plûpart de ses principes, ou obscurs par eux-mêmes, ou énoncés et démontrés d'une maniere obscure, ont-ils donné lieu à plusieurs questions épineuses. [...]

Je me suis proposé [...] de reculer les limites de la Méchanique et d'en applanir l'abord [...] non seulement de déduire les principes de la Méchanique des notions les plus claires, mais de les appliquer aussi à de nouveaux usages; de faire voir tout à la fois, et l'inutilité de plusieurs principes qu'on avoit employés jusqu'ici dans la Méchanique et l'avantage qu'on peut tirer de la combinaison des autres pour le progrès de cette Science; en un mot, d'étendre les principes en les réduisant."

As clearly stated already in his works by Germain, the Principle of Virtual Works supplies the right tool for extending the Cauchy-Navier (see e.g. [10] ) theory of Continuous Bodies.

Actually the Cauchy-Navier format to Continuum Mechanics is not able to encompass the so-called Generalized or Micro-Structured Continua (see e.g. [43], [44], [45], [46], [47], [48], [49] , [76], [87], [55], [103], [81], [120]). These Continua are more and more attracting the interest of many researchers as they seem suitable to supply an efficient description of many phenomena, occurring in continuous systems in which at a "micro" lever are present some sharp variations of physical properties (see e.g. [105], [2]). Also many efforts are directed towards more or less mathematically rigorous homogenization procedures leading to continua which at a "macroscopic" level behave as higher gradient or Cosserat Continua (see e.g. [101], [12], [13], [75], [102], [74], [9], [124], [125], [128], [42], [79], [117]). Homogenization is also involved in the analysis of dissipation effects in many particle systems in the attempt of describing a finite dimensional system (in which length and time scales may be relevant) through a continuum approximation by the Langevin equation in the context of Brownian motion (see e.g. [15],[18], [20]). In these papers, a perturbation analysis is proposed and a procedure for homogenization in stochastic system is found, based on the use of a special family of probability distributions. The resulting continuum models involve constitutive equations, for both the elastic and dissipative behavior, in which memory effects appear: it is an open problem how the treatment developed in the present paper could be adapted to encompass contact interactions which arise in these more complicated models. Based on the original ideas of Lagrange himself, the principles of power balance have received attention also in dynamics, namely in vibrations and acoustics. In this field some authors (see e.g. [16], [17]) attempted to write a self-contained set of equations to describe power migration through a continuum medium: this situation resembles the one in which Dunn and Serrin $([39],[40])$ found themselves in the context of "incomplete" second gradient theories. In the authors' opinion (generalizing what done in [33]) higher gradient theories may complete the cited attempt or, in general, supply a regularised model when non-convex energy functions need to be introduced (as in Cahn-Hilliard and Korteweg fluids, or in many other physical situations, see e.g. [21] or[50]). Finally, the power balance equations can be also approached in the context of uncertainties in the constitutive relationships, where some randomness affects the physical parameters of the continuum (see e.g. [28]). In this case, higher order gradients would be related to the introduction of some statistical 
average and ergodic assumption. Finally, higher order gradient theories are needed when "boundary layer" phenomena must be described: when considering impact phenomena (see e.g. [19], [72], [4] and [6]) in general some "ad hoc" assumptions are imposed, especially when choosing boundary conditions. More detailed models for impact between solids or between solids and fluids, involving some space-time length scales, may cure some of the "singularities" which are present in many models presented in the literature: in particular one could conceive to describe the phenomena of water spray formation or turbulence (see respectively [72] with references there cited or [56]) by means of suitable "contact edge" forces.

In the present paper we show how the Principle of Virtual Powers, as formulated already by E. and F. Cosserat (and in their textbooks by Germain and Salençon) allows us to generalize Cauchy representation formulas for contact interactions to the case of N-th gradient continua.

For this deduction it is crucial to consider the Principle of Virtual Powers valid for every subbody of considered continuous body, as done in all literature based on D'Alembert and Lagrange works (see e.g.[26], [27], [89], [90], [91], [92], [93], [94], [95], [96], [63], [64], [65], [66], [122], [123], [59], [60], [61], [62],[14], [22], [23], [109]). It has to be remarked that, because of a somehow restricted version of this Principle as formulated in [127], pp. 595-600, in many papers its correct formulation has been rediscovered several times.

We will also show that in N-th gradient continua contact interactions:

i) depend in a precise way on the shape of Cauchy Cuts; these cuts will be assumed to be constituted by a finite number of regular faces (orientable and for which all needed gradients of the unit surface normal field exist), a finite number of regular edges (i.e. curves on which are concurring two of the previously introduced faces and for which the Frénet basis and all its needed derivatives exist) and a finite number of wedges (i.e. points in which a finite number of edges are concurring).

ii) must include edge (i.e. concentrated on curves) and wedge (i.e. concentrated on points) interactions, so that one cannot assume (as done in [98], [99], [100]) that these interactions are represented in terms of surface integrals and

iii) cannot reduce simply to forces: indeed(differently from what assumed by $([97]))$ the concept of K-forces (generalizing similar concepts introduced by Rivlin, Mindlin, Green and Germain) is needed.

Indeed, in this paper we prove that "balance of force" is not a suitable postulate for developing the theory of N-th gradient continua.

\section{D'Alembert Principle for $N-$ th gradient Continua}

In this section we start by considering the power of internal interactions and, by means of iterated integrations by parts, we deduce the form of external interactions which can be sustained by $N-t h$ gradient Continua.

\subsection{Power expended by internal or external interactions}

Once we fix a subbody $D$ (i.e. a subset of material particles occupying -in a given configuration- an admissible domain; this last concept is precisely introduced in Appendix A) of a given continuous body $B$ and consider the set $\mathcal{A}$ of all admissible velocity fields for $D$, then it is natural to admit that in $\mathcal{A}$ are included all "test functions" (i.e. infinitely differentiable functions having compact support). In other words, we assume that $\mathcal{D} \subset \mathcal{A}$ where $\mathcal{D}$ denotes the the set of all test functions. It is also natural (as done e.g. in [110] or [59], [60], [61]) to assume that the power expended by internal or external (with respect to $D$ ) interactions is a linear and continuous functional when defined in the set of test functions (with respect to Fréchet topology). In other words we accept the following

Postulate 1 The powers expended by all the interactions relative to a subbody are distributions (in the sense of Schwartz) concentrated on $\bar{D}$, where we denote by $\bar{D}$ the topological closure of the set $D$.

It is clear that, once the previous postulate is accepted, theorems and definitions of the theory of distributions, due to Schwartz (see [111]), become really relevant in continuum mechanics. In particular 
we know that (i) every distribution having compact support $K$ can be represented as the sum of a finite number of derivatives of measures all having their support included in $K$, (ii) a distribution is said to have order smaller than or equal to $k$ if one can represent it as the sum of derivatives with order smaller than or equal to $k$ of measures, (iii) every distribution having support included in a regular embedded submanifold $M$ can be uniquely decomposed as a finite sum of transversal derivatives of extensions of distributions defined on $M$.

Taking into account the aforementioned definitions and theorems, the postulate on the structure of powers can be reformulated in the following way: for every subbody $D$, the powers of both internal and external interactions take the form

$$
\mathcal{P}(D, V)=\int_{\bar{D}}\left(\nabla^{N_{D}} V\right) \mid d T_{D}, \quad \forall V \in \mathcal{D}
$$

where $N_{D}$ is an integer, $d T_{D}$ is a tensor valued measure, and the symbol $\mid$ stands for the total saturation of contravariant and covariant indices.

\subsection{Representations of distributions which are $N$-th order derivatives of absolutely continuous measures concentrated on compact piece-with- boundary smooth manifolds embedded in $\mathbb{R}^{3}$.}

We aim to study some properties of a distribution $\mathcal{L}$ which has support concentrated on a compact piecewith-boundary smooth manifold $M$ (see Appendix A or [71]) embedded in $\mathbb{R}^{3}$ and can be represented as follows

$$
\mathcal{L}(V)=\int_{M} T \mid \nabla^{N} V d \mathcal{H}_{M}
$$

where $T$ is a $N$-times contravariant tensor field of class $C^{N}$ defined on $M$ and $d \mathcal{H}_{M}$ stands for the Hausdorff measure relative to $M$.

We introduce the projector fields $P$ and $Q$ respectively on the tangent and normal subspaces to $M$ (see the Appendix A) and, for any totally symmetric tensor field $T$ of order $N$ defined on $M$, the following "projected" fields

$$
\begin{gathered}
T_{\perp}^{j_{1} \ldots j_{N}}:=T^{i_{1} i_{2} \ldots i_{N}} Q_{i_{1}}^{j_{1}} Q_{i_{2}}^{j_{2}} \ldots Q_{i_{N}}^{j_{N}} . \\
\mathbb{P}(T)^{j_{1} \ldots j_{N}}:=\operatorname{Sym}_{j_{1} \ldots j_{N-1}}\left(\sum_{\alpha=0}^{N-1} C_{N}^{\alpha} T^{i_{1} i_{2} \ldots i_{N}} Q_{i_{1}}^{j_{1}} \ldots Q_{i_{\alpha}}^{j_{\alpha}} P_{i_{\alpha+1}}^{j_{\alpha+1}} \ldots P_{i_{N-1}}^{j_{N-1}}\right) P_{i_{N}}^{j_{N}}
\end{gathered}
$$

where $C_{N}^{\alpha}$ denote the binomial coefficients. We remark that, from the trivial identity $T^{j_{1} \ldots j_{N}}=$ $T^{i_{1} \ldots i_{N}}\left(P_{i_{1}}^{j_{1}}+Q_{i_{1}}^{j_{1}}\right) \ldots\left(P_{i_{N}}^{j_{N}}+Q_{i_{N}}^{j_{N}}\right)$, we can easily derive the equation

$$
\underset{j_{1} \ldots j_{N}}{\operatorname{Sym}} \mathbb{P}(T)^{j_{1} \ldots j_{N}}=\left(T-T_{\perp}\right)^{j_{1} \ldots j_{N}} .
$$

Let us now consider a compact piece-with-boundary smooth manifold $M$ with boundary $\partial M$. Let $T$ be a $C^{1}$ totally symmetric tensor field of order $N$ defined in some neighbourhood of $M$.

Definition 2 We denote by div ${ }_{M}$ the tangential divergence operator on $M$ which associates to any $N-$ th order tensor field $T$ (having components $T^{i_{1} i_{2} \ldots i_{N}}$ ), the tensor field of order $N-1$, div ${ }_{M} T$ (having the components $\left.\left(T^{j_{1} \ldots j_{N-1} l}\right), j_{N} P_{l}^{j_{N}}\right)$. We also introduce the composed operator div $v M$ by setting:

$$
\operatorname{div}_{\nvdash M}(T):=\operatorname{div}_{M}(\mathbb{P}(T))
$$

As usual we define the iterates div $\nvdash_{\nvdash M}^{\alpha}$ of the previous operator in a recursive way by setting

$$
\operatorname{div}_{\nvdash M}^{0} T:=T \quad \text { and } \quad \operatorname{div}_{\nvdash M}^{\alpha}(T):=\operatorname{div}_{\nvdash M}\left(\operatorname{div}_{\nvdash M}^{\alpha-1}(T)\right)
$$


Lemma 3 If $T$ is a totally symmetric $N$ - th order tensor then the following equality holds

$$
\int_{M} T\left|\nabla^{N} V=\int_{M} T_{\perp}\right|\left(\nabla^{N} V\right)_{\perp}-\int_{M} \operatorname{div}_{\nvdash M}(T)\left|\nabla^{N-1} V+\int_{\partial M} \mathbb{P}(T) \cdot \nu\right| \nabla^{N-1} V
$$

where the dot product stands for the saturation of closest contravariant and covariant indices and the vector $\nu$ denotes the tangent vector to $M$, outer pointing unit normal to the boundary $\partial M$. In component representation, formula (8) reads

$$
\begin{aligned}
\int_{M} T^{i_{1} i_{2} \ldots i_{N}} V, i_{1} i_{2} \ldots i_{N} & =\int_{M} T^{i_{1} i_{2} \ldots i_{N}} Q_{i_{1}}^{j_{1}} Q_{i_{2}}^{j_{2}} \ldots Q_{i_{N}}^{j_{N}} V, j_{1} j_{2} \ldots j_{N} \\
& -\int_{M}\left(\left(\mathbb{P}(T)^{j_{1} \ldots j_{N}}\right),_{l} P_{j_{N}}^{l}\right) V,_{j_{1} j_{2} \ldots j_{N-1}} \\
& +\int_{\partial M} \mathbb{P}(T)^{j_{1} \ldots j_{N}} V_{j_{1} j_{2} \ldots j_{N-1}} \nu_{j_{N}}
\end{aligned}
$$

Proof. Using the fact that $\mathbb{P}(T)$ and $\left(T-T_{\perp}\right)$ have the same symmetric part, we can start decomposing the integral

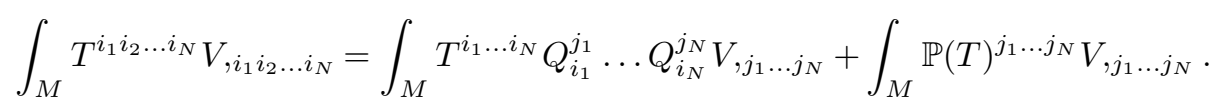

We then note that $\mathbb{P}(T)^{j_{1} \ldots j_{N-1} j_{N}}=\mathbb{P}(T)^{j_{1} \ldots j_{N-1} l} P_{l}^{j_{N}}$ and apply the Divergence Theorem (see Appendix B) on $M$ :

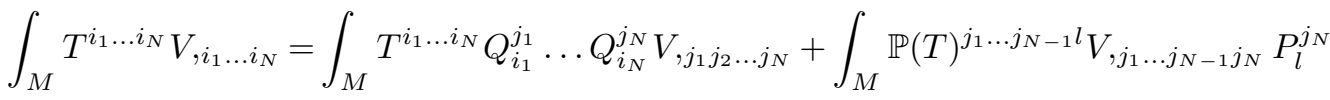

$$
\begin{aligned}
& =\int_{M} T_{\perp}^{j_{1} \ldots j_{N}} V, j_{j_{1} \ldots j_{N}}-\int_{M}\left(\mathbb{P}(T)^{j_{1} \ldots j_{N-1} l}\right), j_{N} P_{l}^{j_{N}} V,{j_{1} \ldots j_{N-1}} \\
& +\int_{M}\left(\mathbb{P}(T)^{j_{1} \ldots j_{N-1} l} V, j_{1} \ldots j_{N-1}\right), j_{N} P_{l}^{j_{N}} \\
& =\int_{M} T_{\perp}^{j_{1} \ldots j_{N}} V{, j_{1} \ldots j_{N}}-\int_{M}\left(\left(\mathbb{P}(T)^{j_{1} \ldots j_{N-1} l}\right), j_{N} P_{l}^{j_{N}}\right) V, j_{1} j_{2} \ldots j_{N-1} \\
& +\int_{\partial M} \mathbb{P}(T)^{j_{1} \ldots j_{N-1} l} V{, j_{1} j_{2} \ldots j_{N-1}}_{l}^{j_{N}} \nu_{j_{N}}
\end{aligned}
$$

which concludes the proof.

Now we can make explicit the announced unique representation of the distribution (2) in terms of transversal derivatives of distributions concentrated on the boundary of $M$. Indeed, applying exactly $N$ times Lemma 3 we can decompose any regular distribution of order $N$ on $M$ in a transverse regular distribution of the same order on $M$ and a distribution of order $N-1$ on $\partial M$ :

Lemma 4 Let $T$ be a $C^{N}$ totally symmetric tensor field of order $N$ defined in some neighbourhood of M. The following equality holds

$$
\begin{aligned}
\int_{M} T \mid \nabla^{N} V=\sum_{J=0}^{N} & (-1)^{N-J} \int_{M}\left(\operatorname{div}_{\nvdash M}^{N-J}(T)\right) \mid\left(\nabla^{J} V\right)_{\perp} \\
& +\sum_{L=0}^{N-1}(-1)^{N-1-L} \int_{\partial M} \mathbb{P}\left(\operatorname{div}_{\nvdash M}^{N-1-L}(T)\right) \cdot \nu \mid \nabla^{L} V .
\end{aligned}
$$


Proof. We proceed by induction. When $N=1$ the statement is a particular case of Lemma 3 . In the inductive step we start assuming that (10) holds for any tensor of order $N$. Then we consider a tensor of order $N+1$. By applying Lemma 3 we get

$$
\int_{M} T\left|\nabla^{N+1} V=\int_{M} T\right|\left(\nabla^{N+1} V\right)_{\perp}-\int_{M} d i v_{\nvdash M} T\left|\nabla^{N} V+\int_{\partial M} \mathbb{P}(T) \cdot \nu\right| \nabla^{N} V
$$

applying the induction assumption to the second term of this expression (where $T$ is replaced by $\operatorname{div} v_{\nvdash} T$ ), we get

$$
\begin{gathered}
\int_{M} T\left|\nabla^{N+1} V=\int_{M} T\right|\left(\nabla^{N+1} V\right)_{\perp}-\sum_{J=0}^{N}(-1)^{N-J} \int_{M}\left(\operatorname{div}_{\nvdash M}^{N-J}\left(\operatorname{div}_{\nvdash M} T\right)\right) \mid\left(\nabla^{J} V\right)_{\perp} \\
-\sum_{L=0}^{N-1}(-1)^{N-1-L} \int_{\partial M} \mathbb{P}\left(\operatorname{div}_{\nvdash M}^{N-1-L}\left(\operatorname{div}_{\nvdash M} T\right)\right) \cdot \nu\left|\nabla^{L} V+\int_{\partial M} \mathbb{P}(T) \cdot \nu\right| \nabla^{N} V .
\end{gathered}
$$

The proof is completed by simply reorganizing the terms.

\section{Principle of Virtual Powers applied to $N$ - th gradient con- tinua.}

The external world can interact with a continuous body $B$. Moreover, its subbodies interact: they exert on each other internal interactions. We underline that, also when considering a given subbody $D$ of $B$ a similar distinction can be done and that some interactions which must be regarded as internal when referring to $B$ actually become external when referring to $D$. We call "internal" and "external" the power expended on any admissible velocity respectively by internal and external interactions.

Definition $5 A$ continuous body $B$ is said to be a $N$ - th gradient continuum if there exists a family of $\Lambda$-times contravariant tensor fields $T_{\Lambda}$ of class $C^{\Lambda}(B)$, such that for every subbody $D \subset B$,

$$
\mathcal{P}^{\text {int }}(D, V)=\sum_{\Lambda=0}^{N} \int_{D} T_{\Lambda} \mid \nabla^{\Lambda} V d L .
$$

The previous definition was put forward by Green and Rivlin who called the tensors $T_{\Lambda}$ the " $\Lambda$-th order stresses". We emphasize that this definition contains strong assumptions : the dependence of the internal power with respect to the subbody is described by a unique measure (the density of internal power) which is absolutely continuous with respect to the Lebesgue measure $d L$ with suitably smooth density.

Note that, in general, because of (1) the fields $T_{\Lambda}$ could be any measures (distributions of order zero). The case of such less regular fields deserves delicate mathematical treatment as done e.g. in [119] and is of great mechanical interest. For instance a power of the form

$$
\mathcal{P}^{\text {int }}(D, V)=\int_{D} \nabla V \mid T d \mathcal{H}_{\left.\right|_{S}}^{2}
$$

where $T d \mathcal{H}_{\left.\right|_{S}}^{2}$ is a tensorial measure concentrated on the surface $S$, can describe an interface with surface tension, an elastic plate or an elastic shell. Such example presents some features which, at first insight, resemble certain properties of contact interactions for smooth stresses which we obtain in the present paper. We attract the attention of the reader to this point in order to prevent a frequent and heavy confusion: indeed in the example given by equation (12) the "stress concentration" on a surface balances the concentration of external forces along the lines constituting $S \cap \partial D$. This phenomenon should not be 
confused with the concentration of external forces obtained in the following formula (14) on any edge of $\partial D$. The first case corresponds to physical concentration of energy (like surface tension or deformation energy of shells) while the second one is related to the geometrical singularity of a Cauchy Cut.

Stating that a continuum is a $N$-th gradient continuum is fundamentally a "constitutive" assumption. By means of it one specifies the order and the smoothness of internal interactions which are considered as "possible" inside the body. The constitutive theory will be completed only when the dependence of the tensors $T_{\Lambda}$ on suitably introduced measures of deformation is specified. For instance, in the Cauchy continuum models which are usually considered, the fundamental constitutive assumption is $N=1$ and $T_{1}$, corresponding to the Cauchy stress tensor, needs to be specified by a further constitutive equation in terms of Green-Saint Venant deformation tensor.

We are now ready to add another postulate

Postulate 6 Principle of Virtual Powers or Power Balance: For every subbody D of a given body $B$ and for every test velocity field $V$ the following equality holds

$$
\mathcal{P}^{i n t}(D, V)=\mathcal{P}^{e x t}(D, V)
$$

Note that in this formulation inertial forces are included in external interactions. For a presentation of the ideas inspiring this axiom we refer to [60], [61], [110] or to the celebrated works [26] and [27]. In these works it is shown that this principle is a generalization of Newton second law which is more suitable when dealing with more general systems than finite systems of material points.

In the present work we will limit ourselves to consider the following class of external interactions:

\section{Postulate 7 Constitutive assumptions for external interactions.}

The external interactions exerted on some subbody $D$ are described by a distribution $\mathcal{P}^{\text {ext }}$ made of two parts. The first part corresponds to long range external interactions exerted on $D$. We assume it to be representable by a distribution which is an integrable function with respect to the Lebesgue measure. The inertial power, which we have included in $\mathcal{P}^{\text {ext }}$, is of this type. The second part corresponds to contact actions. We assume it to be a distribution concentrated on the boundary of D.

\section{Contact actions in $N$-th order strain-gradient continua: $N$-th order forces and stresses.}

A consequence of the Principle of Virtual Powers is that the external contact interactions which a $N-t h$ gradient continuum can "sustain" must belong to a particular subset of the set of distributions concentrated on the boundary of $B$. The sequel of this paper is now devoted to the explicit determination of this particular class. The arguments we will develop follow the same spirit as in e.g [63], [64], [65], [66], [59], [89], [90], [91], [92], [93], [94], [95], [96], [60], [61], [62], [52], [113], [114] and many other papers.

Lemma 8 Let $D$ be a subboby of a $N$ - th gradient continuum B. Let us assume that the topological boundary of $D$ is a compact piece-with- boundary smooth manifolds, then the power of contact interactions are made of three parts: i) a part having support on the faces $\mathcal{S}_{D}$ of the boundary of $D$ and absolutely continuous with respect to the Hausdorff $\mathcal{H}^{2}$ measure. It is a transverse distribution of order smaller or equal to $N-1$, ii) a part having support on the edges $\mathcal{E}_{D}$ of $D$ and absolutely continuous with respect to the $\mathcal{H}^{1}$ measure. It is a transverse distribution of order smaller or equal to $N-2$, iii) a part having support on the wedges $\mathcal{W}_{D}$ of $D$. It is a discrete distribution of order smaller or equal to $N-3$. In 
formulas $^{1}$ :

$$
\begin{aligned}
\mathcal{P}^{\text {ext }}(D, V) & =\int_{\mathcal{D}} E V d \mathcal{H}^{3}+\sum_{\Delta=0}^{N-1} \int_{\mathcal{S}_{D}} F_{\Delta} \mid\left(\nabla^{\Delta} V\right)_{\perp} d \mathcal{H}^{2} \\
& +\sum_{\Delta=0}^{N-2} \int_{\mathcal{E}_{D}} G_{\Delta}\left|\left(\nabla^{\Delta} V\right)_{\perp} d \mathcal{H}^{1}+\sum_{\Delta=0}^{N-3} \int_{\mathcal{W}_{D}} H_{\Delta}\right| \nabla^{\Delta} V d \mathcal{H}^{0}
\end{aligned}
$$

Remark 9 The symbol $\perp$ in the previous formula and in the whole rest of the paper has a contextual sense: it means the transverse part of a tensor relatively to the relevant manifold.

Proof. This result is a consequence of the Principle given in Eq. (13) and of the constitutive assumption of the Postulate 7 . We first apply Lemma 4 to the manifold $D$ and the distribution $\mathcal{P}^{\text {int }}(D, V)$, noticing that the only transverse distributions in $D$ are those of order zero (the normal space at every point in $D$ being reduced to the null space!). We thus obtain a representation for $\mathcal{P}^{i n t}(D, V)$ as the sum of a smooth zero order distribution on $D$ and a $(N-1)-t h$ order distribution concentrated on $\partial D$. Then we apply Lemma 4 to this last distribution and to each face which constitutes $\partial D$. Such distribution is split in a $(N-1)-t h$ order transversal distribution on each face included in $\mathcal{S}_{D}$ and a $(N-2)-t h$ order distribution on each edge included in $\mathcal{E}_{D}$. Finally, we apply Lemma 4 to this $(N-2)-t h$ order distribution and to each one-dimensional manifold with boundary included in $\mathcal{E}_{D}$. Such distribution is split in a $(N-2)-t h$ order transversal distribution on $\mathcal{E}_{D}$ and a $(N-3)-t h$ order distribution on the wedges included in $\mathcal{W}_{D}$ (note that any distribution on wedges is automatically transversal).

The procedure used in this proof actually enables to get a complete representation of contact interactions $\left(E, F_{\Delta}, G_{\Delta}, H_{\Delta}\right)$ in terms of the stress tensors $T_{\Lambda}$. We have decided to postpone this representation since its computation needs the preliminary introduction of a complex notation. We call "contact $\Delta-$ forces" per unit surface, per unit line or concentrated on points, the fields $F_{\Delta}, G_{\Delta}$ and $H_{\Delta}$ following Green and Rivlin or Germain who used this nomenclature only in the case of surface forces.

Let us now explicit the contact $\Delta$-forces $F_{\Delta}, G_{\Delta}$ and $H_{\Delta}$ in terms of the $\Lambda$-order stresses. We start again from the formula (11)

$$
\mathcal{P}^{i n t}(D, V)=\sum_{\Lambda=0}^{N} \int_{D} T_{\Lambda} \mid \nabla^{\Lambda} V d L
$$

and apply Lemma 4 to the manifold $D$ and to every integral in the previous sum. When $M=D$ the projection operator $\mathbb{P}$ is simply the identity, the operator $d i v_{\nvdash D}$ is simply the divergence operator $d i v$ and the outer normal $\nu$ is the outer normal $n$ to the surface $S_{D}$ so that the right hand side of last formula becomes

$$
\sum_{\Lambda=0}^{N}\left(\int_{D}(-1)^{\Lambda}\left(d i v^{\Lambda} T_{\Lambda}\right) V+\sum_{L=0}^{\Lambda-1} \int_{\partial D}(-1)^{\Lambda-1-L}\left(d i v^{\Lambda-1-L} T_{\Lambda}\right) \cdot n \mid \nabla^{L} V\right)
$$

or, reordering,

$$
\int_{D}\left(\sum_{\Lambda=0}^{N}(-1)^{\Lambda} d i v^{\Lambda} T_{\Lambda}\right) V+\sum_{L=0}^{N-1} \int_{\partial D}\left(\sum_{\Lambda=L+1}^{N}(-1)^{\Lambda-1-L}\left(\operatorname{div}^{\Lambda-1-L} T_{\Lambda}\right) \cdot n\right) \mid \nabla^{L} V
$$

\footnotetext{
${ }^{1}$ It is needed here to recall the difference between $\partial D, \partial \partial D$ and $\partial \partial \partial D$ and their support. While $\partial D$ is nothing else than $\mathcal{S}_{D}$ oriented by the external normal to $D$, the situation is more intricate when considering $\partial \partial D$ and $\partial \partial \partial D$. Indeed an edge in $\mathcal{E}_{D}$ coincides with the borders of two regular parts of $\mathcal{S}_{D}$ each one with its own external tangent normal, i.e. a unit vector tangent to the regular part of $\mathcal{S}_{D}$ and normal to the edge. As far as a wedge is concerned many edges can concur at this point defining there as many tangent external vectors, each one tangent to a concurring curve. These definitions are those used generally in the theory of integration of differential forms defined on differential manifolds (for more details see e.g. Arnold (1979)).
} 
This makes natural the introduction of the following notation: we call Bulk Internal Force the quantity

$$
F(D, 0):=\sum_{\Lambda=0}^{N}(-1)^{\Lambda} d i v^{\Lambda}\left(T_{\Lambda}\right)
$$

This quantity depends linearly on the family of stresses $\left\{T_{\Lambda}\right\}$. When needed we will use the notation $\mathfrak{F}\left(D,\left\{T_{\Lambda}\right\}, 0\right):=F(D, 0)$ to recall this dependence. The zero appearing in formula (16) refers to the order of the associated distribution. The dependence with respect to $D$ is here irrelevant but necessary for the coherence with further notation. In the same way we denote $T(D, \Lambda):=T_{\Lambda}$. We also introduce the quantity

$$
T(\partial D, L):=\sum_{\Lambda=L+1}^{N}(-1)^{\Lambda-1-L}\left(\operatorname{div}^{\Lambda-1-L} T_{\Lambda}\right) \cdot n
$$

The expression of internal power is then meaningfully simplified:

$$
\begin{aligned}
\mathcal{P}^{i n t}(D, V) & =\sum_{\Lambda=0}^{N} \int_{D} T(D, \Lambda) \mid \nabla^{\Lambda} V \\
& =\int_{D} F(D, 0) V+\sum_{L=0}^{N-1} \int_{\partial D} T(\partial D, L) \mid \nabla^{L} V
\end{aligned}
$$

We now apply Lemma 4 to any of the regular parts of $\partial D$ in order to treat surface integrals ${ }^{2}$ :

$$
\begin{aligned}
\int_{\partial D} T(\partial D, L) \mid & \nabla^{L} V=\sum_{J=0}^{L}(-1)^{L-J} \int_{\partial D}\left(\left(\operatorname{div}_{\nvdash \partial D}\right)^{L-J} T(\partial D, L)\right) \mid\left(\nabla^{J} V\right)_{\perp \partial D} \\
& +\sum_{J=0}^{L-1}(-1)^{L-1-J} \int_{\partial \partial D} \mathbb{P}_{\partial D}\left(\left(\left(d i v_{\nvdash \partial D}\right)^{L-1-J} T(\partial D, L)\right) \cdot \nu\right) \mid \nabla^{J} V
\end{aligned}
$$

We get for the sum of all surface integrals in (18):

$$
\begin{aligned}
& \sum_{J=1}^{N-1} \int_{\partial D}\left(\sum_{L=0}^{N-1}(-1)^{L-J}\left(\operatorname{div}_{\nvdash \partial D}\right)^{L-J} T(\partial D, L)\right) \cdot\left(\nabla^{J} V\right)_{\perp \partial D} \\
& +\sum_{J=0}^{N-2} \int_{\partial \partial D}\left(\sum_{L=J+1}^{N-1}(-1)^{L-1-J} \mathbb{P}_{\partial D}\left(\left(\operatorname{div}_{\nvdash \partial D}\right)^{L-1-J} T(\partial D, L)\right) \cdot \nu\right) \cdot \nabla^{J} V
\end{aligned}
$$

We continue by introducing new simplifying notation: for any $J \geq 0$, we introduce the surface density of $(J+1)$-forces by setting ${ }^{3}$

$$
F(\partial D, 0):=\sum_{L=0}^{N-1}(-1)^{L}\left(d i v_{\nvdash \partial D}\right)^{L} T(\partial D, L)
$$

and, for any $J>0$,

$$
F(\partial D, J):=\left(\sum_{L=J}^{N-1}(-1)^{L-J}\left(\operatorname{div}_{\nvdash \partial D}\right)^{L-J} T(\partial D, L)\right)_{\perp \partial D} .
$$

\footnotetext{
${ }^{2}$ The reader is warned that we use Cartan notation [7] here (see Appendix A): each integral along an edge corresponds to two integrals coming from the integration by parts on the two faces concurring there. For each of these integrals, the unit tangent normal $\nu$ and the projector $\mathbb{P}_{\partial D}$ are well defined.

${ }^{3}$ The introduced vector quantity $F(\partial D, 0)$ generalizes Cauchy "traction" vector: it is expending power on (virtual) velocities. In Cauchy Continua surface density of force $F(\partial D, 0)$ coincides with the only non-vanishing surface stress $T(\partial D, 0)$. In Second Gradient Continua (as established by Green and Rivlin, Mindlin, Germain) the following equality holds: $F(\partial D, 0)=T(\partial D, 0)-\left(\operatorname{div}_{\partial D} \mathbb{P}_{\delta D}\right) T(\partial D, 1)$.
} 
Clearly all these surface densities depend linearly on the family of stresses $\left\{T_{\Lambda}\right\}$ and, when needed, we recall this dependence by using the notation $\mathfrak{F}\left(\partial D,\left\{T_{\Lambda}\right\}, J\right):=F(\partial D, J)$. We also define the quantities

$$
T(\partial \partial D, J):=\sum_{L=J+1}^{N-1}(-1)^{L-1-J} \mathbb{P}_{\partial D}\left(\left(\operatorname{div}_{\nvdash \partial D}\right)^{L-1-J} T(\partial D, L)\right) \cdot \nu
$$

Then equation 18 becomes:

$$
\begin{aligned}
\mathcal{P}^{i n t}(D, V)= & \int_{D} F(D, 0) V+\sum_{L=0}^{N-1} \int_{\partial D} F(\partial D, L) \mid\left(\nabla^{L} V\right)_{\perp \partial D} \\
& +\sum_{L=0}^{N-2} \int_{\partial \partial D} T(\partial \partial D, L) \mid \nabla^{L} V
\end{aligned}
$$

We must now proceed by applying Lemma 4 to the line integrals in this last formula and to each regular part of $\partial \partial D$. Introducing the projection operator $\mathbb{P}_{\partial \partial D}$ relative to every regular curve composing $\partial \partial D$, and the vector $t_{\partial \partial D}$ denoting the tangent vector of the generic edge concurring in the appropriate wedge the last term in equation (22) becomes

$$
\begin{aligned}
& \sum_{J=0}^{N-2} \int_{\partial \partial D}\left(\sum_{L=J}^{N-2}(-1)^{L-J}\left(\operatorname{div}_{\nvdash \partial \partial D}\right)^{L-J} T(\partial \partial D, L)\right) \mid\left(\nabla^{J} V\right)_{\perp \delta \delta D}+ \\
& +\sum_{H=0}^{N-3} \int_{\partial \partial \partial D}\left(\sum_{L=H+1}^{N-2}(-1)^{L-1-H} \mathbb{P}_{\partial \partial D}\left(\left(d i v_{\nvdash \partial \partial D}\right)^{L-1-H} T(\partial \partial D, L)\right) \cdot t_{\partial \partial D}\right) \mid \nabla^{H} V
\end{aligned}
$$

Introducing once more some simplified notation, we define, for $J \geq 0$ and $H \geq 0$ the line densities of $(J+1)$ - forces $F(\partial \partial D, J)$ and the $(H+1)$-forces concentrated on wedges by setting

$$
F(\partial \partial D, 0):=\sum_{L=0}^{N-2}(-1)^{L}\left(\operatorname{div}_{\nvdash}{ }\right)^{L} T(\partial \partial D, L)
$$

and, for $J>0$ and $H \geq 0$

$$
\begin{aligned}
& F(\partial \partial D, J):=\left(\sum_{L=J}^{N-2}(-1)^{L-J}\left(\operatorname{div}_{\nvdash \partial \partial D}\right)^{L-J} T(\partial \partial D, L)\right)_{\perp \delta \delta D} \\
& T(\partial \partial \partial D, H):=\sum_{L=H+1}^{N-2}(-1)^{L-1-H} \mathbb{P}_{\partial \partial D}\left(\left(d i v_{\nvdash \partial \partial D}\right)^{L-1-H} T(\partial \partial D, L)\right) \cdot t_{\partial \partial D}
\end{aligned}
$$

Remark that, as in every wedge $\mathbb{P}_{\partial \partial \partial D}=0$, then it is necessary to define $F(\partial \partial \partial D, H):=T(\partial \partial \partial D, H)$. Also the newly introduced quantities depend linearly on the family of stresses $\left\{T_{\Lambda}\right\}$. When needed we will recall this dependence by using the notation $\mathfrak{F}\left(\partial \partial D,\left\{T_{\Lambda}\right\}, J\right):=F(\partial \partial D, J) ; \quad \mathfrak{F}\left(\partial \partial \partial D,\left\{T_{\Lambda}\right\}, J\right):=$ $F(\partial \partial \partial D, J)$. By means of (24), (25) and (26) equation (23) becomes

$$
\begin{aligned}
\sum_{L=0}^{N-2} \int_{\partial \partial D} T(\partial \partial D, L) \mid \nabla^{L} V & =\sum_{J=0}^{N-2} \int_{\partial \partial D} F(\partial \partial D, J) \mid\left(\nabla^{J} V\right)_{\perp \delta \delta D} \\
& +\sum_{J=0}^{N-3} \int_{\partial \partial \partial D} F(\partial \partial \partial D, J) \mid \nabla^{J} V
\end{aligned}
$$


The process of integration by parts is ended. We have obtained the searched representation formula for internal power

$$
\begin{aligned}
\mathcal{P}^{i n t}(D, V) & =\int_{D} F(D, 0) V+\sum_{J=0}^{N-1} \int_{\partial D} F(\partial D, J) \cdot\left(\nabla^{J} V\right)_{\perp \delta D} \\
& +\sum_{J=0}^{N-2} \int_{\partial \partial D} F(\partial \partial D, J) \cdot\left(\nabla^{J} V\right)_{\perp \delta \delta D}+\sum_{J=0}^{N-3} \int_{\partial \partial \partial D} F(\partial \partial \partial D, J) \cdot \nabla^{J} V
\end{aligned}
$$

The identification between $\mathcal{P}^{i n t}$ and $\mathcal{P}^{e x t}$ imposed be the Principle 13, the uniqueness of the representation of a distribution in terms of transverse distributions and the just completed calculations lead to the following theorem:

Theorem 10 Representation Theorem for Contact Interactions for N-th Gradient Continua In a $N$ - th gradient continuum as defined in Definition 5, the power of external interactions must take the form (14) where the external interactions $\left(E, F_{J}, G_{J}, H_{J}\right)$ are linearly related to the internal stresses $T_{\Lambda}$ by

$$
\begin{aligned}
& E=\mathfrak{F}\left(D,\left\{T_{\Lambda}\right\}, 0\right) ; \quad F_{J}=\mathfrak{F}\left(\partial D,\left\{T_{\Lambda}\right\}, J\right) \\
& G_{J}=\mathfrak{F}\left(\partial \partial D,\left\{T_{\Lambda}\right\}, J\right) ; \quad H_{J}=\mathfrak{F}\left(\partial \partial \partial D,\left\{T_{\Lambda}\right\}, J\right)
\end{aligned}
$$

and $\mathfrak{F}\left(D,\left\{T_{\Lambda}\right\}, 0\right), \mathfrak{F}\left(\partial D,\left\{T_{\Lambda}\right\}, J\right), \mathfrak{F}\left(\partial \partial D,\left\{T_{\Lambda}\right\}, J\right), \mathfrak{F}\left(\partial \partial \partial D,\left\{T_{\Lambda}\right\}, J\right)$ are given in terms of stresses through the sequence of definitions (16), (17), (19), (20), (21), (24), (25) , (26).

Remark 11 For the highest order forces the representation formulas are simple. Indeed, the tensor $T_{N}$ alone determines the surface $N-1$ forces, the edge $N-2$ forces and the wedge $N-3$ forces as established by means of the following relationships:

$$
\begin{gathered}
\mathfrak{F}\left(\partial D,\left\{T_{\Lambda}\right\}, N-1\right)=\left(T_{N} \cdot n\right)_{\perp \partial D} \\
\mathfrak{F}\left(\partial \partial D,\left\{T_{\Lambda}\right\}, N-2\right)=\left(\mathbb{P}_{\partial D}\left(T_{N} \cdot n\right) \cdot \nu\right)_{\perp \partial \partial D} \\
\left.\left.\mathfrak{F}\left(\partial \partial \partial D,\left\{T_{\Lambda}\right\}, N-3\right)=\left(\mathbb{P}_{\partial \partial D}\left(\mathbb{P}_{\partial D}\left(T_{N} \cdot n\right)\right) \cdot \nu\right)\right) \cdot t_{\partial \partial D}\right)
\end{gathered}
$$

\section{Some final considerations about obtained results}

In this paper we formulate a set of Postulates inspired by the D'Alembertian and Lagrangian vision of Mechanics (suitably adapted to Continuum Mechanics ${ }^{4}$ as envisaged already by E. and F. Cosserat, and, among many others, by P. Germain). The just proven representation Theorem 10 completely solves the problem of determining the dependence of contact interactions on the shape of Cauchy Cuts in N-th gradient continua for the class of Cuts specified in the Appendix A. We want to underline the relevance of some of the obtained results by commenting them in the following subsections.

\subsection{Reconsidering so called "Cauchy Postulate"}

From equations (27) and (28) it is evident the very particular nature of so called "Cauchy Postulate". Many authors had considered Cauchy assumptions about contact interactions as "very" fundamental ones. So fundamental that they refrain from abandoning them and their consequences: most likely it

\footnotetext{
${ }^{4}$ Already Lagrange, in his celebrated and fundamental textbook "Mécanique Analytique" [78], has started this flow of thought. Indeed, one can read at page 195 that the principle of virtual velocities (using Lagrangian nomenclature for the principle of virtual works) must hold for "chacun des corps du systeme proposé" (every body of the considered system). Only two lines later, on the same page, Lagrange deduces the integral formulation of the principle of virtual works valid for "tous les corps du systeme" (all bodies of the system), integral formulation which is the only one considered in [127].
} 
is for this reason that these assumptions are called a "Postulate". They can be resumed as follows: contact interactions are simply represented as surface densities of force and these force fields locally depend only on the unit normal to Cauchy Cuts. The apparently very general Theorem due to [98], (see e.g. also [126]) more deeply rooted the belief that such a Postulate was general enough to encompass all Continuum Mechanics. Actually, as already proven by Green and Rivlin (and clarified by Germain), this is not the case: Noll Theorem assumes that contact interactions concentrated on Cauchy Cuts are absolutely continuous with respect to surface Hausdorff measure, and this property is not verified (see [62]) already by second gradient continua, when Cauchy Cuts include edges (i.e. curves on which the surface normal jumps).

Actually we prove in this paper that the contact force per unit surface at any regular part of a Cauchy cut (what we called face, in the Appendix A) in general does not depend only on the orientation of such surface through its normal $n$. Indeed in $N-t h$ gradient continua such contact force in general depends on the geometry of Cauchy cut as it depends on the gradients of $n$ up to $\nabla^{N-1} n$.

Moreover, contact forces arise on edges and wedges,while K-forces are present on faces, edges and wedges of Cauchy Cuts (see the representation Theorem in the previous section).

\subsection{The concept of Shape of "piece-wise regular" Cauchy Cuts}

Note that Theorem 10, and in particular equation $(28)_{1}$, imply that line contact forces depend in general on the geometry of the curve but also on the geometry of all regular surfaces concurring on the considered edge. In the same way, concentrated interactions which are present at wedges depend on the geometry of edges and faces concurring at this point.

It is clear that the concept of shape of Cauchy Cuts, as introduced e.g. in [32], [33] and in [34], plays a relevant role in the present context.

The results presented in this paper will be generalized, in a forthcoming one, to more general Cauchy Cuts, in which edges where $K$ - th order gradients of the unit normal field may be discontinuous, or where wedges are present at which there are not concurring edges.

More difficult seems the generalization to more singular Cauchy Cuts, although the results of [115], [116], [85], [86], [37], [38], [112] seem to open a possibility in this direction.

\subsection{Cauchy Approach versus D'Alembert Approach}

In [35] it is discussed an alternative approach to the problem solved here. Indeed, instead of starting from a postulated form for internal powers, one could start from a suitable postulate on contact external powers. For second gradient continua this alternative approach, in which the celebrated Cauchy tetrahedron argument is generalized, has been exploited in [32], [33].

Although the two approaches appear to be completely equivalent, the approach "à la Cauchy" is considered more attractive in [127], and consequently second gradient theories have been criticized as they were not originally based on this paradigm.

In a forthcoming paper the aforementioned approach "à la Cauchy" for N-th gradient continua will be presented with some simplifications in the already published proofs: in this way hopefully those who prefer such an approach to Continuum Mechanics will not feel uneasy with $\mathrm{N}$-th gradient Continua.

\subsection{Few considerations about the applicability range of N-th gradient theo- ries}

Continuum Mechanics, if one believes in the atomistic nature of matter, always supplies approximated models for physical systems, in which their "fundamental" discrete nature may be somehow neglected. Actually, Navier obtained the first version of Cauchy continuum theory by means of an average procedure based on an "atomistic" model. Indeed, Cauchy continuum theory can be successfully applied to describe, at a macroscopic level, the behavior of a mechanical system only if it can be considered homogeneous at 
the observation scale and if no microscopic characteristic length scale can influence its overall macroscopic behavior. In every circumstance in which one or more of these length scales are relevant, and in particular when boundary layer phenomena cannot be neglected, then one can keep using continuum theories only if he introduces their more general version treated in the present paper. In the literature there are now many particular physical phenomena described in this spirit (see e.g. (see e.g. [43], [44], [45], [46], [47], [48], [49] , [76], [87], [55], [103], [81], [120], [101], [12], [13], [75], [102], [74], [9], [124], [125], [128], [42], [79], $[117]))$. Here, simply for the particular taste of the authors, it is underlined the possibility of introducing $\mathrm{N}$-th order models for describing some bio-mechanical phenomena ([80], [82],[70], [73], [132]) or those damage phenomena occurring in crack formation and growth (see e.g. [3], [5], [24], [25], [54], [83], [129], $[130])$. 


\section{A Gaussian Geometry of Piecewise Regular Surfaces in $E^{3}$}

The family of subbodies which we will consider in the Principle of Virtual Powers will be open subsets of $C^{*}$ (i.e. the reference configuration of considered body) with a topological boundary which is a piecewise regular surface, as defined in the following section. When regarded as embedded submanifolds, actually the topological boundaries we consider are compact piece-with-boundary smooth manifolds (see [71]). These surfaces represent a particular class of "shapes" as introduced in [32], [33].

\section{A.1 Piecewise regular surfaces embedded in $E^{3}$.}

In the present paper we will use the following nomenclature (following the fundamental textbook [1]):

Definition 12 A surface $S$ is a piecewise regular (orientable and rectifiable) surface embedded in $E^{3}$ when there exist a finite set

$$
\left\{\gamma_{i} \subset S, i=1, \ldots K\right\}
$$

of $C^{1}$ curves (called edges) and a finite set of points (called wedges)

$$
\left\{W_{i} \in S, i=1, \ldots, H\right\}
$$

such that, once introduced the notation (which is reminiscent of the one usually encountered in the formulation of Poincaré theorem for exterior forms)

$$
\begin{aligned}
\text { Support }(\partial S) & :=\left(\bigcup_{i=1}^{K} \gamma_{i}\right) ; \\
\text { Support }(\partial \partial S) & :=\left(\bigcup_{i=1}^{H} W_{i}\right),
\end{aligned}
$$

the conditions appearing in the following list are verified.

\section{LIST OF CONDITIONS}

- for every $p \in S-(\partial S \cup \partial \partial S)$ (i.e. for every regular point belonging to $S)$ there exists a neighbourhood in $S$ which is locally $\left(C^{2}-\right)$ diffeomorphic to $\mathbb{R}^{2}$ : we call any such local diffeomorphism

$$
r: \mathbb{R}^{2} \rightarrow S-(\partial S \cup \partial \partial S)
$$

an internal chart of $S$, We assume that for every internal chart $r$ the set $r\left(\mathbb{R}^{2}\right)$ is a rectifiable surface;

- for every $p \in S-(\partial S \cup \partial \partial S)$ there exists a translation vector in $E^{3}$, denoted with the symbol $n(p)$, which is orthogonal to every tangent vector to $S$, (the field $n(\cdot)$ is assumed to be suitably smooth in any internal chart of $S$ ).

- for every $p \in \partial S-\partial \partial S$ there exist two diffeomorphisms (called also "border charts")

$$
r^{ \pm}:\left[0, \infty\left[\times \mathbb{R} \rightarrow I^{ \pm} \subset S\right.\right.
$$

such that

$$
\begin{gathered}
r^{ \pm}(0,0)=p ; \quad r^{ \pm}(0, \mathbb{R})=\partial S \cap I^{ \pm} \\
(\forall y \in] 0, \infty[\times \mathbb{R})\left(r^{ \pm}(y) \in S-(\partial S \cup \partial \partial S)\right)
\end{gathered}
$$

and both the following limits exist

$$
\lim _{x \rightarrow(0,0)} n\left(r^{ \pm}(x)\right):=n^{ \pm}(p) .
$$


Therefore every curve $\gamma_{i}$ can be regarded as the border of two regular surfaces $S^{ \pm}$one on the side + the other on the side - with respect to $\gamma_{i}$. We will denote the unit outward pointing normal vector to $\gamma_{i}$ in the tangent plane to $S^{ \pm}$respectively with the symbol $\nu^{ \pm}$.

- for every curve $\gamma_{i}$ (the length of which is denoted by $l_{i}$ ) there exists a global parametric $C^{1}$ representation $r_{i}$

$$
r_{i}: s \in\left[0, l_{i}\right] \mapsto p \in S
$$

such that

$$
\left\|\frac{d r_{i}}{d s}\right\|=1, \quad \frac{d r_{i}}{d s} \cdot n^{ \pm}=0
$$

We will assume that

$$
\frac{d r_{i}}{d s} \times n^{ \pm}= \pm \nu^{ \pm}
$$

- for every $W_{j} \in \partial \partial S$ there exists at least one curve $\gamma_{i}$ such that one of the two following conditions holds

$$
r_{i}(0)=W_{j} \text { or } r_{i}\left(l_{i}\right)=W_{j}
$$

Roughly speaking piecewise regular orientable and rectifiable surfaces, in the particular conventional sense specified above, are surfaces where the normal vector is defined in all points except those belonging to a finite set of wedges and to a finite set of regular curves (the edges of the surface). Along these curves a tangent vector is always defined together with both the normals of the two subsurfaces concurring on the edge.

Definition 13 We call face of $S$ every connected component of the set $S-(\partial S \cup \partial \partial S)$

\section{A.2 Local parametrizations for regular curves and surfaces. Local curvilinear coordinate systems in $E^{3}$ adapted to surfaces and surface edges.}

We start recalling some basic definitions from differential geometry (see e.g. [118])

Definition 14 A parametrization of a regular curve is a $C^{1}$ one-to-one function $\left.r:\right] a_{1}, b_{1}\left[\rightarrow E^{3}\right.$ such that

$$
\left(\forall p \in r(] a_{1}, b_{1}[)\right)\left(t(p):=\frac{d r}{d x^{1}}\left(r^{-1}(p)\right) \neq 0\right) .
$$

The vector $t(p)$ is the tangent vector to the curve in $p$.

Definition 15 At every point $p$ of a regular curve $\gamma$ we can define the following projection operators

$$
P_{\gamma, p}:=t(p) \otimes t(p) ; \quad Q_{\gamma, p}:=n_{1}(p) \otimes n_{1}(p)+n_{2}(p) \otimes n_{2}(p)
$$

where $n_{1}(p)$ and $n_{2}(p)$ form an independent set of vectors both orthogonal to $t(p)$. When this will not cause confusion we will skip one or both the indices of the introduced projectors. $P_{\gamma, p}$ will be called the projector in the tangent bundle (or line) of $\gamma$ at point $p$, while $Q_{\gamma, p}$ will be called the projector in the orthogonal bundle (or plane) of $\gamma$ at point $p$.

Definition 16 A map

$$
\left.r:\left(x^{1}, x^{2}\right) \in\right] a_{1}, b_{1}[\times] a_{2}, b_{2}\left[\subset \mathbb{R}^{2} \mapsto r\left(x^{1}, x^{2}\right) \in E^{3}\right.
$$

is called a local parametrization for the regular surface $S$ in the neighborhood of $p$ if $r$ is a $C^{1}$ diffeomorphism between $] a_{1}, b_{1}[\times] a_{2}, b_{2}\left[\right.$ and $r(] a_{1}, b_{1}[\times] a_{2}, b_{2}[)=: I_{p}$ such that

$$
\left.I_{p} \subset S ; \quad r^{-1}(p) \in\right] a_{1}, b_{1}[\times] a_{2}, b_{2}[
$$


Once a local parametrization for the regular surface $S$ is introduced then a set of coordinate curves on $S$ is established, together with a field of bases for the tangent planes to $S$.

We will consider in the following the fields of vectors induced by a local parametrization

$$
(\forall \alpha \in\{1,2\})\left(\forall q \in I_{p}\right)\left(a_{\alpha}(q):=\frac{\partial r}{\partial x_{\alpha}}\left(r^{-1}(q)\right)\right) .
$$

The couple $\left\{a_{1}(q), a_{2}(q)\right\}$ is a basis of the tangent plane to $S$ in the point $q$. The set of points

$$
r\left(\left\{x^{1}\right\} \times\right] a_{2}, b_{2}[), \quad r(] a_{1}, b_{1}\left[\times\left\{x^{2}\right\}\right)
$$

are called the coordinate $x^{2}$ and $x^{1}$ curves. Remark that the vectors $a_{\alpha}$ are tangent to the coordinate $x_{\alpha}$ curves. The $C^{1}$ normal (to $S$ ) unit vector field $n$ can be calculated by the formula

$$
n(q)=\frac{a_{1}(q) \times a_{2}(q)}{\left\|a_{1}(q) \times a_{2}(q)\right\|}
$$

Definition 17 Let the couple $\left\{a_{1}(p), a_{2}(p)\right\}$ denote an othonormal basis of the tangent plane to $S$ in the point $p$. At every point $p$ of a regular surface $S$ we can define the projector operators

$$
P_{S, p}:=a_{1}(p) \otimes a_{1}(p)+a_{2}(p) \otimes a_{2}(p) ; \quad Q_{S, p}:=n(p) \otimes n(p) .
$$

When this will not cause confusion we will skip one or both the indices of the introduced projectors. $P_{S, p}$ will be called the projector in the tangent bundle (or plane) of $S$ at point $p$, while $Q_{S, p}$ will be called the projector in the orthogonal bundle (or line) of $S$ at point $p$.

Remark 18 Because of the previous definitions, regular curves and surfaces are respectively one dimensional and two dimensional manifolds embedded in the three dimensional Euclidean space.

The scalar fields

$$
g_{\alpha \beta}: q \in I_{p} \mapsto a_{\alpha}(q) \cdot a_{\beta}(q)
$$

represent the components of a tensor field which is called the Riemannian metric induced on $S$ by the inner product in $E^{3}$.

Remark 19 A given regular curve or surface can be endowed with the structure of Riemannian manifold simply using the inner product of the Euclidean space in which they are embedded. Indeed for any couple $v$ and $w$ of vectors in their tangent bundle one can calculate their inner product simply by regarding them as vectors in $E^{3}$.

Although in the Euclidean space $E^{3}$ the Cartesian system of coordinates, using at every point the same vector basis to represent displacement vectors, is in general sufficient, in the present context one needs to introduce the following

Definition 20 Local curvilinear coordinate systems in $E^{3}$. A map

$$
\left.\varphi:\left(x^{1}, x^{2}, x^{3}\right) \in\right] a_{1}, b_{1}[\times] a_{2}, b_{2}[\times] a_{3}, b_{3}\left[\subset \mathbb{R}^{3} \mapsto \varphi\left(x^{1}, x^{2}, x^{3}\right) \in E^{3}\right.
$$

is called a local chart in $E^{3}$ in the neighbourhood $I_{p}$ of $p \in E^{3}$ determining a local curvilinear coordinate system when it is a diffeomorphism between

$$
] a_{1}, b_{1}[\times] a_{2}, b_{2}[\times] a_{3}, b_{3}\left[\text { and } \varphi(] a_{1}, b_{1}[\times] a_{2}, b_{2}[\times] a_{3}, b_{3}[)=: I_{p}\right.
$$

A $i-$ th $(i \in\{1,2,3\})$ coordinate curve is obtained fixing in the function $\varphi$ all arguments except the $x_{i}$ variable, and the tangent vectors of such coordinate curves are denoted as follows

$$
\left(\forall q \in I_{p}\right)\left(a_{i}(q):=\frac{\partial \varphi}{\partial x_{i}}\left(\varphi^{-1}(q)\right)\right) .
$$

Obviously for every $q \in I_{p}$ the set $\left\{a_{i}(q), i \in\{1,2,3\}\right\}$ is a basis of the vector space of translations in $E^{3}$. Therefore the curvilinear coordinate system which has been introduced generates a field of bases in all $I_{p}$. 
Definition 21 In $I_{p}$ we can introduce the following scalar fields

$$
\left(\forall q \in I_{p}\right)\left(g_{i j}(q):=a_{i}(q) \cdot a_{j}(q)\right)
$$

which are the components, in considered curvilinear coordinate system, of the Riemann metric in the Euclidean field $E^{3}$.

Let us consider a piecewise regular surface: as we will see in what follows, it is possible to introduce in the neighbourhood of $p$ charts (i.e. curvilinear coordinate systems) in the Euclidean space $E^{3}$ which are "adapted" to i) the surface in the neighbourhood of regular point $p \in S-(\partial S \cup \partial \partial S)$ ii) to the edge of the surface in the neighbourhood of a point which is not a wedge.

\section{A.2.1 Local curvilinear coordinate system in $E^{3}$ adapted to $S$ in the neighbourhood of a regular point.}

The following Lemma is a consequence of the Inverse Function Theorem and is the basis of an important part of Gaussian differential geometry (for a proof see e.g. [31], [77]).

Lemma 22 Let $r$ be a local parametrization of $S$ in the neighbourhood of the regular point $p \in S-(\partial S \cup$ $\partial \partial S)$. For a suitably small positive $\varepsilon$ the map $\varphi$ defined by

$$
\begin{aligned}
\varphi & \left.:\left(x^{1}, x^{2}, x^{3}\right) \in\right] a_{1}, b_{1}[\times] a_{2}, b_{2}[\times]-\varepsilon,+\varepsilon\left[\subset \mathbb{R}^{3}\right. \\
& \mapsto r\left(x^{1}, x^{2}\right)+x^{3} n\left(r^{-1}\left(x^{1}, x^{2}\right)\right) \in E^{3}
\end{aligned}
$$

actually is an invertible function and a diffeomorphism.

Definition 23 When the map $\varphi$ considered in the previous Lemma is a diffeomorphism then it is called the chart adapted to $S$ induced by the parametrization $r$.

When this will not be cause of confusion we will admit an abuse of notation and we will use the symbols $n\left(x^{1}, x^{2}\right), g_{\alpha \beta}\left(x^{1}, x^{2}\right)$ and $a_{\alpha}\left(x^{1}, x^{2}\right)$ instead of the symbols $n\left(r^{-1}\left(x^{1}, x^{2}\right)\right), g_{\alpha \beta}\left(r^{-1}\left(x^{1}, x^{2}\right)\right)$ and $a_{\alpha}\left(r^{-1}\left(x^{1}, x^{2}\right)\right)$. The same abuse of notation will be repeated for all the fields $a_{i}$ and $g_{i j}$ not specifying the composition with the function $\varphi^{-1}$. The class of charts we have now introduced was first introduced by Gauss (see e.g. [118]). We are now able to extend in the neighbourhood in $E^{3}$ of a regular point $p \in S$ the fields of projectors in the tangent and in the orthogonal bundles.

Definition 24 Let us consider a chart $\varphi$ adapted to the surface $S$ in the neighbourhood of a point p. For every $\left.x_{3} \in\right]-\varepsilon, \varepsilon\left[\right.$ we can consider the (regular) surface $S_{x_{3}}$ which is defined by the following local parametrization

$$
r_{x_{3}}:=r+x_{3} n .
$$

On every surface $S_{x_{3}}$ it is easy to introduce the tangent and orthogonal projectors, which for $x_{3}=0$ reduce to the projectors introduced already for $S=S_{0}$. We will denote these fields of projectors, also when defined in the opens set

$$
\varphi(] a_{1}, b_{1}[\times] a_{2}, b_{2}[\times]-\varepsilon,+\varepsilon[) \subset E^{3}
$$

with the same symbols $P_{S, p}$ and $Q_{S, p}$.

\section{A.2.2 Local curvilinear coordinate system in $E^{3}$ adapted to an edge of $S$ in the neighbour- hood of a point which is not a wedge.}

Let $r$ be a local parametrization of an edge $\gamma$ of $S$ in the neighbourhood of a point $p \in \partial S-\partial \partial S$. When it is a diffeomorphism, we will call the maps $\varphi^{ \pm}$defined by

$$
\begin{aligned}
\varphi^{ \pm} & \left.:\left(x^{1}, x^{2}, x^{3}\right) \in\right] a_{1}, b_{1}[\times]-\varepsilon,+\varepsilon[\times]-\varepsilon,+\varepsilon\left[\subset \mathbb{R}^{3}\right. \\
& \mapsto r\left(x^{1}\right)+x^{2} n^{ \pm}\left(r^{-1}\left(x^{1}\right)\right)+x^{3} \nu^{ \pm}\left(r^{-1}\left(x^{1}\right)\right) \in E^{3}
\end{aligned}
$$


a chart adapted to $S$ at the considered edge as induced by the edge parametrization $r$ on the side + or - (respectively) depending on the consistent choice adopted. Adapting the argument used in the proof of the Lemma of the previous subsection, and using the assumed regularity hypotheses about $S$ and its edges, it can be proven again that, when the value of $\varepsilon$ is chosen to be positive and suitably small, $\varphi^{ \pm}$ actually are two diffeomorphisms and can be used as charts in $E^{3}$.

Definition 25 Let us consider a regular point of the edge $\gamma$ and a chart adapted to $S$ at $\gamma$. For every $\left.\left(x^{2}, x^{3}\right) \in\right]-\varepsilon,+\varepsilon[\times]-\varepsilon,+\varepsilon[$ we can consider the regular curves parametrized by the function

$$
r_{x^{2}, x^{3}}^{ \pm}:=r+x^{2} n^{ \pm}+x^{3} \nu^{ \pm}
$$

For each of these curves we can define the projection on the orthogonal and tangent bundles, thus obviously extending in the neighbourhood of $\gamma$ the already introduced projection fields $P_{\gamma, p}$ and $Q_{\gamma, p}$.

\section{B Gauss Divergence Theorem for embedded Riemannian man- ifolds}

We choose a global orthonormal basis $\left(e_{i}, i=1,2,3\right)$ for the vector field of displacements in $E^{3}$. All tensor fields, unless differently specified, will be represented by means of the components with respect this basis. In this section we consider an embedded Riemannian manifold $M$ in $E^{3}$. This manifold can be therefore a regular curve or surface. Because we were able to construct the so-called Gaussian coordinate systems adapted to considered manifolds, then in a whole neighbourhood of these manifolds it is possible to introduce the projection operator fields $P$ and $Q$. For reducing the complication of the calculation which we will perform in what follows we do not use directly the adapted curvilinear coordinates: instead, after having established the existence of the fields $P$ and $Q$ in the neighbourhood of $M$, we introduce a global Cartesian coordinate system and represent all fields in it. This technical choice is exactly the same one which allowed to Germain the generalization, for second gradient materials, of the results found by Green, Rivlin, Toupin and Mindlin.

It is easy to prove the following:

Lemma 26 If on each manifold $M, P$ denotes the projection on the tangent bundle : we have

$$
\begin{array}{r}
\delta_{i}^{j}=P_{i}^{j}+Q_{i}^{j}, \quad P_{i}^{j} P_{j}^{k}=P_{i}^{k}, \\
Q_{i}^{j} Q_{j}^{k}=Q_{i}^{k}, \quad P_{i}^{j} Q_{j}^{k}=0 .
\end{array}
$$

The unit external normal to $M$ on its border is denoted $\nu$; it belongs to the tangent space to $M$.

Using these notations the divergence theorem reads (see e.g. [118])

Theorem 27 For any vector field $W$ defined in the neighbourhood of $M$

$$
\int_{M}\left(P_{j}^{i} W^{j}\right)_{, k} P_{i}^{k}=\int_{\partial M} W^{i} P_{i}^{k} \nu_{k}
$$

This theorem together with relation

$$
Q_{j, k}^{i} P_{i}^{k}=-Q_{j}^{i} P_{i, k}^{k}
$$

implies that 
Corollary 28 For any vector field $W$ defined in a neighbourhood of $M$

$$
\begin{aligned}
\int_{M}\left(W^{i}\right){ }_{k} P_{i}^{k}=\int_{M}\left(P_{j}^{i} W^{j}\right)_{, k} P_{i}^{k}+\left(Q_{j}^{i} W^{j}\right)_{, k} P_{i}^{k}= \\
=\int_{M} W^{j} Q_{j, k}^{i} P_{i}^{k}+\int_{\partial M} W^{i} P_{i}^{k} \nu_{k}= \\
=-\int_{M} W^{j} Q_{j}^{i} P_{i, k}^{k}+\int_{\partial M} W^{i} P_{i}^{k} \nu_{k} .
\end{aligned}
$$

\section{References}

[1] R. Abraham, J.E. Marsden, and T. Ratiu, Manifolds,Tensor Analysis, and Applications. Applied Mathematical Sciences, 75, Springer Verlag, (1988).

[2] J.-J. Alibert, P. Seppecher, F. dell'Isola, Truss modular beams with deformation energy depending on higher displacement gradients. Math. Mech. Solids, 8:1 (2003) 51-73.

[3] U. Andreaus, P. Baragatti, Cracked beam identification by numerically analysing the nonlinear behaviour of the harmonically forced response. J. Sound Vibration, 330:4 (2011) 721-742.

[4] U. Andreaus, L. Placidi, G. Rega, Numerical simulation of the soft contact dynamics of an impacting bilinear oscillator. Communications in Nonlinear Science \& Numerical Simulation, 15:9 (2010) 26032616.

[5] U. Andreaus, P. Baragatti, Fatigue crack growth, free vibrations and breathing crack detection of Aluminium Alloy and Steel beams. Journal of Strain Analysis for Engineering Design, 44:7 (2009) 595-608.

[6] U. Andreaus, L. Placidi and G. Rega, Soft impact dynamics of a cantilever beam: equivalent SDOF model versus infinite-dimensional system. Proc. IMechE Vol. 225 Part C: J. Mechanical Engineering Science, 225:10 (2011) 2444-2456.

[7] V.I. Arnold, Mathematical Methods of Classical Mechanics. Springer Verlag (1979)-(1989).

[8] C. Banfi, A. Marzocchi and A. Musesti, On the principle of virtual powers in continuum mechanics. Ricerche di Matematica, 55 (2006) 299-310.

[9] S. Bardenhagen, N. Triantafyllidis, Derivation of higher order gradient continuum theories in 2,3-D nonlinear elasticity from periodic lattice models. J. Mech. Phys. Solids, 42:1 (1994) 111-139 .

[10] E. Benvenuto, La scienza delle costruzioni e il suo sviluppo storico. Sansoni, Firenze, (1981).

[11] V. Berdichevsky, Variational Principles of Continuum Mechanics. Springer, (2009).

[12] F. Bouyge, I. Jasiuk, and M. Ostoja-Starzewski, A micromechanically based couple-stress model of an elastic two-phase composite. Int. Journal Solids Structures 38:10-13 (2001) 1721-1735.

[13] F. Bouyge, I. Jasiuk, S. Boccara and M. Ostoja-Starzewski, A micromechanically based couplestress model of an elastic orthotropic two-phase composite. Eur. J. Mech./A: Solids 21:3 (2002) 465-481.

[14] J.L. Bleustein, A note on the boundary conditions of Toupin's strain-gradient theory. Int. J. Solids Struct., 3:6 (1967) 1053-1057.

[15] A. Carcaterra, A. Akay, Dissipation in a finite-size bath. Phys. Rev. E 84, 011121 (2011). 
[16] A. Carcaterra, Ensemble energy average and energy flow relationships for nonstationary vibrating systems. J. Sound Vibration, 288:3 (2005) 751-790.

[17] A. Carcaterra, A. Sestieri, Energy Density Equations and Power Flow in Structures. J. Sound Vibration, 188:2 (1995) 269-282.

[18] A. Carcaterra, A. Akay, I.M. Ko, Near-irreversibility in a conservative linear structure with singularity points in its modal density. J. Acoust. Soc. Am., 119:4 (2006) 2141-2149.

[19] A. Carcaterra, E. Ciappi, A. Iafrati, E.F. Campana, Shock spectral analysis of elastic systems impacting on the water surface. J. Sound Vibration, 229:3 (2000), 579-605.

[20] A. Carcaterra, A. Akay, Theoretical foundations of apparent-damping phenomena and nearly irreversible energy exchange in linear conservative systems. J. Acoust. Soc. Am., 121:4 (2007) 19711982 .

[21] P. Casal, et H.Gouin, Relation entre l'équation de l'énergie et l'équation du mouvement en théorie de Korteweg de la capillaritè. C. R. Acad. Sci. Paris, t. 300, Série II, N. 7 (1985) 231-233.

[22] P. Casal, La théorie du second gradient et la capillarité. C. R. Acad. Sci. Paris, t. 274, Série A (1972) 1571-1574.

[23] P. Casal, La capillarité interne. Cahier du

[24] A. Chambolle, G. A. Francfort, and J.-J. Marigo, Revisiting energy release rates in brittle fracture. Journal of Nonlinear Science 20 (2010) 395-424.

[25] A. Chambolle, G. A. Francfort, and J.-J. Marigo, When and how do cracks propagate. J. Mech. Phys. Solids, 56 (2009) 16141622.

[26] E. Cosserat and F. Cosserat, Note sur la théorie de l'action euclidienne. Paris, Gauthier-Villars, (1908).

[27] E. Cosserat, and F. Cosserat, Sur la Théorie des Corps Déformables, Herman, Paris, (1909).

[28] A. Culla, A. Sestieri, A. Carcaterra, Energy flow uncertainties in vibrating systems: Definition of a statistical confidence factor. Mechanical Systems and Signal Processing, 17:3 (2003) 635-663.

[29] N. Daher, G.A.Maugin, Virtual power and thermodynamics for electromagnetic continua with interfaces. J. Math. Phys. 27:12, (1986) 3022-3035. Maugin2011

[30] N. Daher, G.A. Maugin, The method of virtual power in continuum mechanics. Application to media presenting singular surfaces and interfaces. Acta Mech. 60:3-4 (1986) 217-240.

[31] F. dell'Isola and W. Kosinski, Deduction of thermodynamic balance laws for bidimensional nonmaterial directed continua modelling interphase layers. Archives of Mechanics, 45 (1993) 333-35.

[32] F. dell'Isola and P. Seppecher, The relationship between edge contact forces, double force and interstitial working allowed by the principle of virtual power. C. R. Acad. Sci. - Serie IIb: Mecanique, Physique, Chimie, Astronomie, vol. 321 (1995) 303-308.

[33] F. dell'Isola and P. Seppecher, Edge Contact Forces and Quasi-Balanced Power. Meccanica, 32 (1997) 33-52.

[34] F. dell'Isola, P. Seppecher, A. Madeo, Beyond Euler-Cauchy Continua: The structure of contact actions in N-th gradient generalized continua: a generalization of the Cauchy tetrahedron argument. CISM Lecture Notes C-1006, Chap.2, Springer Verlag (2011). 
[35] F. dell'Isola, P. Seppecher, A. Madeo, Fluid Shock Wave Generation at Solid-Material Discontinuity Surfaces in Porous Media, CISM Lecture Notes C-1006, Chap.7, Springer Verlag (2011).

[36] A. Di Carlo e A. Tatone, (Iper-)Tensioni \& Equi-Potenza. AIMETA'01 XV Congresso AIMETA di Meccanica Teorica e Applicata 15th AIMETA Congress of Theoretical and Applied Mechanics 2001.

[37] M. Degiovanni, A. Marzocchi and A. Musesti,Edge-force densities and second-order powers. Annali di Matematica, 185 (2006) 81-103.

[38] M. Degiovanni, A. Marzocchi, and A. Musesti, Cauchy fluxes associated with tensor fields having divergence measure. Arch. Ration. Mech. Anal., 147 (1999) 197-223.

[39] J.E. Dunn, and J. Serrin, On the thermomechanics of interstitial working. Arch. Rational Mech. Anal., 88:2 (1985) 95-133.

[40] J.E. Dunn, Interstitial working and a non classical continuum thermodynamics. In: J. Serrin (Ed), New Perspectives in Thermodynamics, Springer Verlag, Berlin, pp. 187-222 (1986).

[41] G.E. Exadaktylos, I. Vardoulakis, Microstructure in linear elasticity and scale effects: a reconsideration of basic rock mechanics and rock fracture mechanics. Tectonophysics, 335:1-2 (2001) 81-109.

[42] A.C. Fannjiang, Y.S. Chan, and G.H. Paulino, Strain gradient elasticity for antiplane shear cracks: a hypersingular integrodifferential equation approach. SIAM J. Appl. Math, 62:3, (2001) 1066-1091.

[43] S. Forest, M. Amestoy, S. Cantournet, G. Damamme, S. Kruch, Mécanique des Milieux Continus, ECOLE DES MINES DE PARIS Année 2005-2006.

[44] S. Forest, Mechanics of generalized continua: construction by homogenization. J.Phys. IV France, 8 (1998) Pr4 39-Pr4 48.

[45] S. Forest, Homogenization methods and the mechanics of generalized continua - part 2. Theoretical and applied Mechanics 28-29 (2002) 113-143.

[46] S. Forest, Milieux continus généralisés et matériaux hétérogènes. Les Presses de l'Ecole des Mines de Paris, ISBN : 2-911762-67-3, (2006).

[47] S. Forest, Generalized continua. In K.H.J. Buschow, R.W. Cahn, M.C. Flemings, B. Ilschner, E.J. Kramer, and S. Mahajan, editors, Encyclopedia of Materials : Science and Technology updates, pp. 1-7. Elsevier, Oxford, (2005).

[48] S. Forest and M. Amestoy, Mécanique des milieux continus. Cours de l'Ecole des Mines de Paris n $3121,(2004,2005,2006)$.

[49] S. Forest, Milieux continus généralisés et matériaux hétérogènes. Mémoire d'habilitation à diriger des recherches, (2004).

[50] A. Forestier, S. Gavrilyuk, Criterion of hyperbolicity for non-conservative quasilinear systems admitting a partially convex conservation law. Math. Meth. Appl. Sci., 3:1 (2011) 21482158.

[51] R. Fosdick, E.G. Virga, A variational proof of the stress theorem of Cauchy. Arch.Ration.Mech.Anal. 105 (1989) 95-103.

[52] R. Fosdick, Observations concerning virtual power. Math.Mech.Solids 16 (2011) 573-585.

[53] R. Fosdick, On the principle of virtual power for arbitrary parts of a body. Continuum Mech. Thermodyn, 23 (2011) 483-489. 
[54] G. A. Francfort, J.-J. Marigo, Revisiting brittle fracture as an energy minimization problem. J. Mech. Phys. Solids, 46:8 (1998) 1319-1342.

[55] S. Gavrilyuk and H. Gouin, A new form of governing equations of fluids arising from Hamilton's principle. Int. J. Engineering Science, 37 (1999) 1495-1520.

[56] S. Gavrilyuk, H. Gouin, Geometric evolution of the Reynolds stress tensor in three-dimensional turbulence. A. Greco, S. Rionero, T. Ruggeri (editors), World Scientific, (2010) 182-190, ISBN 978-981-43170-41-2.

[57] E. Fried and M.E. Gurtin, Tractions, balances, and boundary conditions for nonsimple materials with application to liquid flow at small length scales. Arch. Rational Mech. Analysis 182 (2006) 513-554.

[58] E. Fried and M.E. Gurtin, A continuum mechanical theory for turbulence: a generalized Navier -Stokes-equation with boundary conditions. Theoretical and Computational Fluid Dynamics, 182 (2008) 513-554.

[59] P. Germain, Cours de Mècanique des Milieux Continus, tome I. Masson, Paris, (1973).

[60] P.Germain, La méthode des puissances virtuelles en mécanique des milieux continus. Première partie. Théorie du second gradient. J. Mécanique 12 (1973) 235-274.

[61] P. Germain, The method of virtual power in continuum mechanics - Part 2: Microstructure. SIAM J. Appl. Math. 25 (1973) 556-575.

[62] P. Germain, Sur l'application de la méthode des puissances virtuelles en mécanique des milieux continus. C. R. Acad. Sci. Paris Série A-B, 274 A1051-A1055 (1972).

[63] A.E. Green, R. S. Rivlin, Multipolar continuum mechanics. Arch. Rational Mech. Anal, 17 (1964) 113-147.

[64] A.E. Green, R. S. Rivlin, Simple force and stress multipoles. Arch. Rational Mech. Anal., 16 (1964) 325-353.

[65] A.E. Green, R. S. Rivlin, On Cauchy's equations of motion. Z. Angew. Math. Phys. (ZAMP) 15 (1964) 290-292.

[66] A.E. Green, R. S. Rivlin, Multipolar continuum mechanics: Functional theory I. Proc. Roy. Soc. Ser. A, 284 (1965) 303-324.

[67] M.E. Gurtin, A gradient theory of single-crystal viscoplasticity that accounts for geometrically necessary dislocations. Int. J. Plasticity 50 (2002) 809-819.

[68] M.E. Gurtin, L.Anand, Thermodynamics applied to gradient theories involving the accumulated plastic strain: the theories of Aifantis and Fleck and Hutchinson and their generalization. J. Mech. Phys. Solids 57 (2009) 405-421.

[69] M.E. Gurtin, E. Fried, L. Anand, The Mechanics and Thermodynamics of Continua. Cambridge University Press, Cambridge (2009).

[70] E. Hamed, Y. Lee, I. Jasiuk, Multiscale modeling of elastic properties of cortical bone Acta Mechanica 213:(1-2) (2010) 131-154.

[71] J.H. Hubbard, B.B. Hubbard, Vector Calculus, Linear algebra and differential forms, a unified approach. Prentice Hall ed., (2009). 
[72] A. Iafrati, A. Carcaterra, E. Ciappi, E.F. Campana, Hydroelastic Analysis of a Simple Oscillator Impacting the Free Surface. Journal of Ship Research 44:3-4 (2000) 278-289.

[73] I. Jasiuk, Modeling of trabecular bone as a hierarchical material. Computational Fluid and Solid Mechanics 1:2 (2003) 1727-1728.

[74] I. Jasiuk and M. Ostoja-Starzewski, On the reduction of constants in planar Cosserat elasticity with eigenstrains and eigencurvatures. J. Thermal Stresses 26 (2003) 1221-1228.

[75] H. Joumaa and M. Ostoja-Starzewski, Stress and couple-stress invariance in non-centrosymmetric micropolar planar elasticity, online,Proceedings of Royal Society A (2011).

[76] N. Kirchner, P. Steinmann, On the material setting of gradient hyperelasticity (English summary). Math. Mech. Solids, 12:5 (2006) 559-580.

[77] W. Kosinski, Field Singularities and Wave Analysis in Continuum Mechanics. Ellis Horwood Series: Mathematics and Its Applications, Wiley and Sons, PWN - Polish Scientific Publishers, Warsaw (1986).

[78] J.-L. Lagrange, Mécanique Analytique. Éditions Jaques Gabay 1989, Sceaux.

[79] R. Larsson, S. A Diebels, Second-order homogenization procedure for multi-scale analysis based on micropolar kinematics. Internat. J. Numer. Methods Engrg. 69:12 (2007) 2485-2512.

[80] T. Lekszycki and F. dellIsola, A mixture model with evolving mass densities for describing synthesis and resorption phenomena in bones reconstructed with bio-resorbable materials. Accepted by ZAMM Journal of Applied Mathematics and Mechanics / Zeitschrift fur Angewandte Mathematik und Mechanik.

[81] J. Li and M. Ostoja-Starzewski, Micropolar continuum mechanics of fractal media. Int. J. Eng. Sci., 49 (2011) 1302-1310.

[82] A. Madeo, T. Lekszycki and F. dellIsola, A continuum model for the bio-mechanical interactions between living tissue and bio-resorbable graft after bone reconstructive surgery. CRAS Mécanique, 339: 10 (2011) 625-682.

[83] J.-J. Marigo, C. Pideri, The effective behavior of elastic bodies containing microcracks or micro holes localized on a surface, International Journal of Damage Mechanics, 20 (2011) 1151-1177.

[84] M. Lazar, G. A. Maugin, A note on line forces in gradient elasticity. Mechanics Research Communications, 33 (2006) 674-680.

[85] A. Marzocchi, A.Musesti, Balanced virtual powers in Continuum Mechanics. Meccanica 38 (2003) 369-389.

[86] A. Marzocchi, A.Musesti, Decomposition and integral representation of Cauchy interactions associated with measures. Cont. Mech. Thermodyn., 13 (2001) 149-169.

[87] G.A.Maugin, A.V. Metrikine, Mechanics of Generalized Continua, One Hundred Years After the Cosserats, Springer (2010).

[88] G.A. Maugin, The principle of virtual power: from eliminating metaphysical forces to providing an efficient modelling tool In memory of Paul Germain (1920-2009). Cont. Mech. Thermodyn. Published On-Line DOI 10.1007/s00161-011-0196-7, pp. 1-20.

[89] R.D. Mindlin, Second gradient of strain and surface tension in linear elasticity. Int. J. Solids and Struct. 1:4 (1965) 417-438. 
[90] R.D. Mindlin, Micro-structure in linear elasticity. Arch. Rat. Mech. Analysis 16 (1964) 51-78.

[91] R.D. Mindlin, Complex representation of displacements and stresses in plane strain with couplestresses. 1965 Appl. Theory of Functions in Continuum Mechanics (Proc. Internat. Sympos ., Tbilisi), Vol. I, Mechanics of Solids (Russian) pp. 256-259 Izdat. Nauka”, Moscow (1963).

[92] R.D. Mindlin, H.F.Tiersten, Effects of couple-stresses in linear elasticity. Arch. Rational Mech. Anal., 11 (1962) 415-448 .

[93] R.D. Mindlin, Influence of couple-stresses on stress concentrations Main features of cosserat theory are reviewed by lecturer and some recent solutions of the equations, for cases of stress concentration around small holes in elastic solids, are described. Experimental Mechanics, 3:1 (1962) 1-7.

[94] R.D. Mindlin, N.N. Eshel, On first strain-gradient theories in linear elasticity. International Journal of Solids and Structures, 4:1 (1968) 109-124.

[95] R.D. Mindlin, Stress functions for a Cosserat continuum. Int. J. Solids Structures, 1:3 (1965) 265271.

[96] R.D.Mindlin, On the equations of elastic materials with micro-structure. Int. J. Solids Structures, 1:1 (1965) 73-78.

[97] W. Noll, E.G.,Virga, On edge interactions and surface tension. Arch. Rational Mech. Anal., 111:1 (1990) 1-31.

[98] W. Noll, The foundations of classical mechanics in the light of recent advances in continuum mechanics. Proceeding of the Berkeley Symposium on the Axiomatic Method, Amsterdam, pp. 226-281 (1959).

[99] W. Noll, Lectures on the foundations of continuum mechanics and thermodynamics. Arch. Rational Mech. Anal., 52 (1973) 62-92.

[100] W. Noll, The geometry of contact separation and reformation of continuous bodies. Arch. Rational Mech. Anal., 122:3 (1993) 197-212.

[101] M. Ostoja-Starzewski, S. Boccara and I. Jasiuk, Couple-stress moduli and characteristic length of composite materials. Mechanics Research Communications 26:4 (1999) 387-396.

[102] M. Ostoja-Starzewski and I. Jasiuk, Stress invariance in planar Cosserat elasticity. Proceedings of the Royal Society, London A 451, 453-470; Errata 452, 1503, (1995).

[103] M. Ostoja-Starzewski, Macrohomogeneity condition in dynamics of micropolar media. Arch. Appl. Mech. 81:7 (2011) 899-906.

[104] M. Ostoja-Starzewski, Microstructural Randomness and Scaling in Mechanics of Materials, Chapman \& Hall/CRC Press, (2009).

[105] C. Pideri and P. Seppecher, A second gradient material resulting from the homogenization of an heterogeneous linear elastic medium. Contin. Mech. Thermodyn. 9:5 (1997) 241-257.

[106] P. Podio-Guidugli, A virtual power format for thermomechanics. Continuum Mech. Thermodyn., 20 (2009) 479-487.

[107] P. Podio-Guidugli, Contact interactions, stress, and material symmetry, for nonsimple elastic materials. (English, Serbo-Croatian summary) Issue dedicated to the memory of Professor Rastko Stojanovic (Belgrade, 2002). Theoret. Appl. Mech. 28/29 (2002) 261-276. 
[108] P. Podio-Guidugli and M. Vianello, Hypertractions and hyperstresses convey the same mechanical information. Continuum Mech. Thermodyn. 22 (2010) 163-176.

[109] C. Polizzotto, Strain-gradient elastic-plastic material models and assessment of the higher order boundary conditions. Eur. J. Mech. A Solids, 26:2 (2007) 189-211.

[110] J. Salençon, Mécanique des milieux continus. Ed. Ellipses (1988)-(1995), Handbook of Continuum Mechanics, Ed. Springer (Berlin, 2001) Mécanique des milieux continus. Tome I. Éd. École polytechnique, Palaiseau ; Ellipses, Paris, (2002)-(2005).

[111] L. Schwartz, Théorie des Distributions, Hermann Paris, (1973).

[112] F. Schuricht, A new mathematical foundation for contact interactions in continuum physics. Arch. Ration. Mech. Anal. 184 (2007) 495-551.

[113] P. Seppecher, Etude des conditions aux limites en théorie du second gradient: cas de la capillarité. C. R. Acad. Sci. Paris, t. 309, Série II 497502 (1989).

[114] P. Seppecher, Etude d'une Modélisation des Zones Capillaires Fluides: Interfaces et Lignes de Contact. Thèse de l'Université Paris VI, Avril (1987).

[115] M. Šilhavý, The existence of the flux vector and the divergence theorem for general Cauchy fluxes. Arch.Ration. Mech. Anal., 90 (1985) 195-211.

[116] M. Šilhavý, Cauchy's stress theorem and tensor fields with divergences in Lp. Arch. Ration. Mech. Anal., 116 (1991) 223-255.

[117] M. Sokolowski, Theory of couple-stresses in bodies with constrained rotations. In CISM courses and lectures, vol. 26 Berlin, Germany: Springer (1970).

[118] M. Spivak, A comprehensive introduction to differential geometry, Vol. I and II. Second edition. Publish or Perish, Inc., Wilmington, Del. (1979).

[119] P. Steinmann, On Boundary Potential Energies in Deformational and Configurational Mechanics. J. Mech. Phys. Solids 56 (2008) 772-800.

[120] A.S.J. Suiker and C.S. Chang, Application of higher-order tensor theory for formulating enhanced continuum models. Acta Mech., 142 (2000) 223-234.

[121] R. Sunyk, P. Steinmann, On Higher Gradients in Continuum-Atomistic Modelling. Int. J. Solids Structures 40:24 (2003) 6877-6896.

[122] R.A. Toupin, Elastic Materials with couple-stresses. Arch. Rat. Mech. Analysis, 11 (1962) 385-414.

[123] R.A. Toupin, Theories of elasticity with couple-stress.Arch. Rational Mech. Anal., 17 (1964) 85-112.

[124] N. Triantafyllidis and S. Bardenhagen, On higher order gradient continuum theories in 1-D nonlinear elasticity. Derivation from and comparison to the corresponding discrete models. J. Elasticity, 33:3 (1993) 259-293.

[125] N. Triantafyllidis and S. Bardenhagen, The influence of scale size on the stability of periodic solids and the role of associated higher order gradient continuum models. J. Mech. Phys. Solids, 44:11 (1996) 1891-1928.

[126] C.A. Truesdell, A First Course in Rational Continuum Mechanics, Vol. I General Concepts. Academic Press, New York, (1977). 
[127] C. Truesdell, R.A. Toupin, The Classical Field Theories, Handbuch der Physik III/1. Springer, Berlin (1960).

[128] N. Triantafyllidis and E.C. Aifantis, A gradient approach to localization of deformation. I. Hyperelastic materials. J. Elasticity, 16:3 (1986) 225-237.

[129] Y.Yang and A. Misra, Higher-order stress-strain theory for damage modeling implemented in an element-free Galerkin formulation. Computer Modeling in Engineering and Sciences, 64:1 (2010) $1-36$.

[130] Y. Yang, W.Y. Ching and A. Misra, Higher-order continuum theory applied to fracture simulation of nano-scale intergranular glassy film. Journal of Nanomechanics and Micromechanics, 1:2 (2011) 60-71.

[131] G. Vailati, Il principio dei lavori virtuali da Aristotele a Erone d'Alessandria. Scritti (Bologna, Forni, 1987), vol. II, pp. 113-128, Atti della R. Accademia delle Scienze di Torino, vol. XXXII, adunanza del 13 giugno 1897, quaderno IG (091) 75 I - III (1897).

[132] C. Y. Wang, L. Feng, I. Jasiuk, Scale and Boundary Conditions Effects on the Apparent Elastic Moduli of Trabecular Bone Modeled as a Periodic Cellular Solid. J. Biomechanical Eng.Transactions of the ASME, 12 (2009) 131. 